\title{
Measuring the performance of peer-to-peer systems with social networks characteristics.
}

\begin{abstract}
In social system, people with similar interests gather and create a community. This structure organizes people effectively and makes sharing information easier. In sociology the behavior of such structures has been investigated for a long time. Fortunately this structure can be extended to Peer-to-Peer (P2P) systems. This is due to the fact that peers in P2P systems usually have few interests like people in the real world and they try to find other peers with similar interests. On the other hand, the structure of the underlying models in P2P has a direct effect on different aspect of such systems. In this study the performance related parameters of a P2P system with social network characteristics are measured by simulation. The result shows that using similar structure as same as real world inside a community produces better performance. In addition, flooding technique in such systems creates higher traffic than random structured model; however a simple controlled flooding can provide a satisfaction result.
\end{abstract}

Keyword: Distributed systems; Overlay network; Community; Model. 